symptoms. Stigma and social isolation T-scores were above the general population average $(\mathrm{T}$-score $=50)$ in 69 $(58.5 \%)$ and $57(48.3 \%)$ participants, respectively. Higher levels of both stigma and social isolation correlated with more severe depression $(=0.527, \mathrm{p}<0.0001$ and $=0.551$, $\mathrm{p}<0.0001$, respectively). Social isolation correlated with stigma $(=0.945, \mathrm{p}<0.0001)$ and the effect of stigma on depression was no longer significant $(=-0.018, \mathrm{p}=0.88)$ after we examined the mediator effect of social isolation and controlled for sociodemographics (table 1, Model 1). Lower education ( high school vs some college or higher) significantly increased the strength of correlation between stigma and depression after controlling for sociodemographics (table 1, Model 2). Poverty and African American race also increased the strength of the relationship but were not significant.

Conclusions Nearly a third with CCLE reported moderate to severe depressive symptoms. Social stigma contributed to depression through feelings of social isolation, and those with lower education were more vulnerable to the impact of stigma on depression. Clinical and public health programs should help strengthen social connections in people with CCLE and reduce stigmatization in the community, particularly among those from socioeconomically disadvantaged groups.

Funding Source(s): Centers for Disease Control and Prevention (CDC) grant U01DP005119.

\section{VALIDATION OF PEDANAM AS AN INSTRUMENT OF COGNITIVE EVALUATION IN NEUROPSYCHIATRIC SLE}

${ }^{1}$ Jaqueline de Amorim* ${ }^{*}{ }^{2}$ Simone Kishimoto, ${ }^{3}$ Paula Fernandes, ${ }^{4}$ Clovis Silva, ${ }^{3}$ Roberto Marini, ${ }^{5}$ Simone Appenzeller. ${ }^{1}$ Child and Adolescente graduate program UNICAMP; ${ }^{2}$ Physiopathology graduate program; ${ }^{3}$ UNICAMP; ${ }^{4}$ Instituto da Criança USP; ${ }^{5}$ University of Campinas

\subsection{6/lupus-2019-Ism.243}

Background Translation, validation, and use of a computerized battery for cognitive evaluation of patients with systemic lupus erythematosus (SLE) as part of an investigation of neuropsychiatric manifestations is useful and allows multicenter studies.

Methods This is a quantitative transversal study with control group conducted in pediatric and adult rheumatology clinics. The computer test batteries ANAM (Automated Neuropsychological Assessment Metrics) or PedAnam (Pediatric version) was submitted to the Guidelines for the Process of Cross-Cultural Adaptation. This process has five stages: Initial translation, synthesis of the translations, back translation, harmonization, test of the prefinal version and final version. After the process, we apply the ANAM in 98 SLE patients and healthy age-matched individuals. All individuals underwent an evaluation through the battery tests taking $30 \mathrm{~min}$ on average to solve the problems.

Results 98 SLE patients, 69 female and 29 male, ages between 6 and 68 years with mean of 28.6. The control group had 84 people, 73 female and 11 male, ages between 6 and 65 years with mean of 25 . For the evaluation was used the Performance Validity Index score, that provides a performance indicator, between 0 and 14, for someone with good effort, or above for someone outside the range of that expected for someone providing good effort. Patients presented an average performance of 8.05 , with a minimum of 0 and a maximum of 33 , while the control group had a mean of 4.4 , minimum 0 and maximum of 27 . As the smaller score results in a better effort, it is possible to notice meaningful differences between the groups $(\mathrm{p}<0.05)$.

Conclusions Cognitive difficulties are often observed in SLE and practical tools like ANAM and PedAnan should be used to measure the cognitive loss that patients may have; these losses should be monitored more closely if there are other neuropsychiatric symptoms.

Funding Source(s): CNPq

CAPES

FAPESP

\section{POLYMORPHISM OF MICRORNA REGION IN THE TNFA GENE IN CHILDHOOD-ONSET SYSTEMIC LUPUS ERYTHEMATOSUS}

${ }^{1}$ Jessica Vivaldo*, ${ }^{1}$ Mariana Postal, ${ }^{1}$ Nailu Sinicato, ${ }^{1}$ Roberto Marini, ${ }^{2}$ Timothy Niewold, ${ }^{3}$ Simone Appenzeller. ' UNICAMP; ${ }^{2}$ NYU; ${ }^{3}$ University of Campinas

\subsection{6/lupus-2019-Ism.244}

Background Studies have implicated microRNAs (miRNAs) in the pathogenesis of systemic lupus erythematosus (SLE). miRNAs regulate approximately $90 \%$ of the protein-encoding genes and play a central role in various biological processes, including impairment of cell differentiation and proliferation e apoptosis. Polymorphism in miRNA regions of TNFA gene may account for the variations observed in the clinical. The aim of this study was to investigate the presence of polymorphisms of miRNA regions of TNFA gene associated to clinical and laboratory profile of these SLE patients.

Methods Consecutive childhood-onset SLE (cSLE) patients followed at Pediatric Rheumatology Unit of the Unicamp were enrolled in study. Healthy volunteers with were included as control group. A complete clinical, laboratory and neurological was performed in all subjects. cSLE patients were further assessed for clinical and laboratory SLE manifestations, disease activity [SLE Disease Activity Index (SLEDAI)], damage [Systemic Lupus International Collaborating Clinics/American College of Rheumatology Damage Index (SDI)] and current therapy. Total dose of corticosteroids and other immunosuppressant medications used since the onset of disease were calculated by data obtained by careful review of the medical charts. We investigated miRNA region of the TNFA gene in cSLE compared to healthy volunteers using DNA Sequencing by Capillary Electrophoresis. Data were compared by nonparametric tests.

Results We included 110 cSLE patients [83 women $(75.4 \%)]$. The mean disease duration was $13.18 \pm 4.32$ (120 years). We included as a control group 60 healthy individuals [52 women $(86.7 \%)$ ] recruited from the local community. The mean score of cumulative SLEDAI was 2.88 $\pm 2.20(0.09-12.52)$. The mean of total corticosteroid dose was $23437.54 \pm 16656.55 \mathrm{mg}$. We identified polymorphism rs3093665 (c. $* 77 \mathrm{~A}>\mathrm{C}$ ) in 8 (7.3\%) cSLE patients, polymorphism rs3093666 (c.*419 C>T) in 3 (2.7\%) patients and polymorphism rs3093667 (c. $* 453 \mathrm{G}>\mathrm{T}$ ) in $2(1.8 \%)$ cSLE patients. Polymorphism rs3093665 is located in a region of interest, into a miRNA region-binding site of miR-452 and polymorphism rs3093667 into a miRNA 
region-binding site of miR-19a. In the control group, we identified polymorphism rs3093665 in only 1 control. We observed an association between polymorphism rs3093665 and organic brain syndrome $(\mathrm{p}=0.001)$, an association between polymorphism rs3093666 and thrombocytopenia $(\mathrm{p}=0.02)$ and an association between polymorphism rs3093667 and hematuria $(p=0.024)$.

Conclusions To our knowledge, this is the first study to evaluate polymorphisms in miRNA regions of TNFA gene in SLE. We identified polymorphism rs3093665 and rs 3093667, both into miRNA region-binding sites. Alterations contribute to the development of pathological conditions and clinical disorders in SLE.

Funding Source(s): CAPES

$\mathrm{CNPq}$

FAPESP

\section{ASSOCIATIONS BETWEEN PATIENT ACCEPTABLE SYMPTOM STATEAND THREE DOMAINS OF THE DISEASE IN SLE: A CROSS-SECTIONAL STUDY OF 1,364 PATIENTS FROM THE SPANISH SOCIETY OF RHEUMATOLOGY LUPUS REGISTRY} ${ }^{4}$ Javier Narváez-García, ${ }^{5}$ Francisco Javier López-Longo, ${ }^{6}$ Maria Galindo Izquierdo, ${ }^{7}$ Jaime Calvo Alen, ${ }^{8}$ Antonio Fernandez Nebro, ${ }^{5}$ Juan Ovalles-Bonilla, ${ }^{9}$ Paula Rubio Muñoz, ${ }^{10}$ Eva Tomero Muriel, ${ }^{3}$ Coral Mouriño Rodriguez, ${ }^{11}$ Esther Uriarte Isacelaya, ${ }^{12}$ Angela Pecondón Español, ${ }^{13}$ Mercedes Freire González, ${ }^{14}$ Ricardo Blanco Alonso, ${ }^{15}$ Marian Gantes Mora, ${ }^{16}$ Monica Ibañez Barcelo, ${ }^{17}$ Carlos Montilla Morales, ${ }^{3}$ Jose Maria Pego Reigosa. 'Hospital Universitario Lucus Augusti; ${ }^{2}$ Hospital de Gran Canaria DR. Negrín; ${ }^{3}$ Complexo hospitalario Universitario Vigo; ${ }^{4}$ Hospital de Bellvitge; ${ }^{5}$ Hospital General Universitario Gregorio Marañón; ${ }^{6}$ Hospital Universitario 12 de Octubre; ${ }^{7}$ Hospital Universitario Araba; ${ }^{8}$ UGC de Reumatología, Instituto de Investigación Biomédica de Málaga (IBIMA) Hospital Regional Universitario de Málaga, Spain; ${ }^{9}$ Hospital Germans Trias I Pujol; ${ }^{10} \mathrm{Hospital}$ de la Princesa; ${ }^{11} \mathrm{Hospital}$ de Donostia; ${ }^{12} \mathrm{Hospital}$ Miguel Servet; ${ }^{13} \mathrm{Hospital}$ Juan Canalejo A Coruña (CHUAC); ${ }^{14}$ Hospital Marques de Valdecilla; ${ }^{15}$ Hospital Universitario de Canarias; ${ }^{16}$ Hospital Son Llàtzer de Palma de Mallorca; ${ }^{17}$ Hospital Clinico Universitario Salamanca

10.1136/lupus-2019-Ism.245
Background In a large cohort of systemic lupus erythematosus (SLE) patients, to evaluate the association between a Patient Acceptable Symptom State (PASS) and three different domains of the disease: activity, damage and impact of SLE on patients lives.

Methods Cross-sectional study of 1,364 SLE (ACR 97 Criteria) patients from the Spanish Society of Rheumatology Lupus Registry (RELESSER). Patients were clinically assessed by the physicians, who collected data about demographics, clinical manifestations, laboratory features and therapies. SLE activity was assessed by using the SLEDAI (SLE Disease Activity Index). The Systemic Lupus International Collaborating Clinics/American College of Rheumatology Damage Index (SLICC/ACR DI) was used to measure damage accrual. The impact of SLE on patients lives was evaluated by using the Lupus Impact Tracker (LIT). Finally, the PASS question 'Considering all the different ways your disease is affecting you, if you would stay in this state for the next months, do you consider that your current state is satisfactory? was answered in a dichotomized way: yes/no. Measures of central tendency and spread were used to describe responses and T-test was used to evaluate the independence between PASS and the other questionnaires and indices.

Results Mean age (years) \pm S.D. of the 1364 patients included in the study was $49.9 \pm 13.3 ; 90.3 \%$ were female and $94.9 \%$ were Caucasian. In the total of patients, 979 (71.8\%) were in PASS. Patients in PASS had significantly lower activity (SLEDAI Score) than those not in PASS $(2.4 \pm 3.2$ vs. $3.0 \pm 3.9$, respectively; $\mathrm{p}=0.007)$. The SLICC/ACR DI was significantly lower in the group of patients in PASS compared to those without PASS $(0.64 \pm 1.1$ vs. $0.81 \pm 0.21$, respectively; $\mathrm{p}=0.022$ ). SLE had a significantly lower impact on patients in PASS in comparison to those without PASS (LIT Score: 8.47 \pm 7.5 vs. $19.8 \pm 8.34$, respectively; $\mathrm{p}=0.001$ ). Distribution and statistics for each group are shown in table 1.

Conclusions In the largest observational European Registry of SLE patients, PASS was associated with other domains of the disease: activity, damage and impact on patients life. PASS is a simple and reliable patient-reported outcome that can be a useful tool to evaluate patients well-being.

Funding Source(s): None

\begin{tabular}{|c|c|c|c|c|c|c|}
\hline \multicolumn{7}{|c|}{ Abstract 245 Table 1} \\
\hline & & $n$ & Mean & $\pm \mathrm{SD}$ & $P$ value & Cohen's d size effect \\
\hline \multirow[t]{2}{*}{ SLEDAI } & PASS yes & 969 & 2.40 & 3.27 & \multirow[t]{2}{*}{0.007} & \multirow[t]{2}{*}{$0,18(0,06-0,30)$} \\
\hline & PASS no & 380 & 3.02 & 3.96 & & \\
\hline \multirow[t]{2}{*}{ SLICC ACR } & PASS yes & 969 & 0.64 & 1.10 & \multirow[t]{2}{*}{0.01} & \multirow[t]{2}{*}{$0.15(0.03-0.27)$} \\
\hline & PASS no & 380 & 0.81 & 1.21 & & \\
\hline \multirow[t]{2}{*}{ LIT } & PASS yes & 962 & 8.47 & 7.53 & \multirow[t]{2}{*}{0.001} & \multirow[t]{2}{*}{$1.46(1.33-1.59)$} \\
\hline & PASS no & 375 & 19.81 & 8.34 & & \\
\hline
\end{tabular}

